\title{
Aspektoppositionen und Aktionsarten im Französischen
}

\author{
Franz-Josef KLein \\ Romanische Sprachwissenschaft, Universität Siegen, Deutschland
}

Romanistikken er ikke den disciplin, hvor kategorierne ,aspekt' og , aktionsart' er blevet udviklet eller spiller en dominerende rolle. Alligevel er tematikken, der er benævint med disse termini, relevant også for de romanske sprog. I følgende bidrag gælder det for mig på den ene side at fremstille den specifikt romanistiske forskningstradition om begreberne 'aspektoppositioner' og 'aktionsarter'. På den anden side, og først og fremmest, skal der fremhæves nogle perspektiver for den fremtidige forskning med henblik på aktionsart-problematikken.

1.

In der linguistischen Literatur, ganz gleich, auf welche Sprachen sie sich bezieht, wurde und wird das Thema ,Aspekt' vielfach im Zusammenhang mit der Problematik der ,Aktionsarten' behandelt. ${ }^{1}$ Zeitweise wurden die beiden Termini geradezu synonym verwendet. Erst allmählich bildete sich ein Konsens dahingehend heraus, dass es sinnvoll ist, die besagten Kategorien auf sprachliche Erscheinungen unterschiedlicher Ebenen anzuwenden. Von entscheidender Wirkung wurde hierbei ein Vorschlag des schwedischen Linguisten Sigurd Agrell, der im Jahre 1908 dafür plädierte, ,Aspekt' und ,Aktionsart' in der folgenden Weise voneinander abzugrenzen:
„Die beiden Hauptkategorien des slawischen Zeitwortes, die unvollendete und die vollendete Handlungsform (das Imperfektivum und das Perfektivum), [...] nenne ich Aspekt [...] Mit dem Ausdruck Aktionsart bezeichne ich [...] Bedeutungsfunktionen der Verbalkomposita (sowie einiger Simplizia und Suffixbildungen), die genauer ausdrücken, wie die Handlung vollbracht wird, die Art und Weise ihrer Ausführung markieren." 2

Der Terminus ,Aspekt' bezeichnet dieser Abgrenzung nach also ein morphologischgrammatisches Phänomen, der Ausdruck ,Aktionsart' dagegen bleibt dem Bereich der Lexikologie (speziell der Wortbildung und der Semantik) vorbehalten. Die terminologische Unterscheidung Agrells hat aber, wie allgemein bekannt ist, erhebliche Schwierigkeiten gehabt, sich durchzusetzen. In der linguistischen Literatur herrschte, was das Verhältnis von ,Aspekten' und ,Aktionsarten' angeht, dementsprechend noch lange Zeit beträchtliche Verwirrung. ${ }^{3}$ Diese resultierte weniger aus der (legitimen) Frage, ob eine strikte Trennung der beiden Kategorien überhaupt sinnvoll ist. Maßgeblich für 
die entstandene Situation war vielmehr die Tatsache, dass die beiden Termini vielfach unzureichend definiert wurden. ${ }^{4}$ Die unvermeidliche Konsequenz war, dass sowohl der Terminus ,Aspekt' als auch der Ausdruck ,Aktionsart' unreflektiert auf grammatische ebenso wie auf lexikalische Erscheinungen angewandt wurden. Auch in der heutigen Linguistik werden die beiden Termini nicht immer klar voneinander getrennt. ${ }^{5}$

In der Romanistik, um die es hier in erster Linie gehen soll, verhielt es sich, was den Umgang mit den beiden Kategorien angeht, nicht anders als in den übrigen Philologien. ,Aspekt' und ,Aktionsart' wurden (und werden) hier terminologisch nicht immer klar geschieden. Zuweilen wurden die beiden Phänomene selbst miteinander verwechselt, manchmal wurden sie bewusst gleichgesetzt. In der französisch-, spanischoder italienischsprachigen Romanistik mag dies teilweise dadurch mit bedingt sein, dass die Dichotomie ,Aspekt'/'Aktionsart' nicht ohne weiteres in die romanischen Sprachen übersetzbar ist. Die beiden Termini werden undifferenziert mit frz. ,aspect', span. ,aspecto', ital. ,aspetto' usw. wiedergegeben. Der einzige mir bekannt gewordene, französisch schreibende Romanist, der eine explizite terminologische Unterscheidung versucht, ist der Tscheche Otto Ducháček. Er übersetzt ,Aktionsart' ebenso wörtlich wie schwerfällig mit ,caractère de l'action' ${ }^{6}$ Aber auch in der deutschsprachigen Romanistik, in der die Unterscheidung von ,Aspekt' und ,Aktionsart' sprachlich möglich war, wurden die beiden Termini noch lange Zeit undifferenziert verwendet. Als Beispiel mag etwa die 1930 erschienene, wichtige Monographie Die Aktionsarten im Französischen von Walter Hanckel dienen. ${ }^{7}$ Diese scheint im Titel auf die lexikalische Thematik zu referieren; de facto behandelt das Buch jedoch zu großen Teilen grammatische Fragen, die nach Agrells Definition eindeutig in den Bereich der Aspektoppositionen gehören. Den entscheidenden Durchbruch hin zu mehr terminologischer Klarheit brachte in der Romanistik Wolfgang Pollaks 1960 erschienenes Buch Studien zum, Verbalaspekt' im Romanischen (Pollak 1960). Pollak, seinerzeit Professor für Romanische Philologie an der Universität Wien, reserviert - ganz im Sinne Sigurd Agrells - den Terminus ,Aktionsart' für die Ebene der lexikalischen Bedeutung, während er im ,Aspekt' ein grammatisches Phänomen sieht:

„Aktionsarten modifizieren die lexikalische Bedeutung des

Verbs, der Aspekt ist eine rein syntaktische Kategorie." ${ }^{\text {.8 }}$

Die Aktionsart eines Wortes manifestiert sich nach Pollak im isolierten Infinitiv; es handelt sich somit um eine kontextunabhängige Gegebenheit. Dagegen stellt die aspektbedingte Perfektivierung ein kontextuell bedingtes Phänomen dar, durch das die „Bedeutungssubstanz des Verbums nicht modifiziert“ wird (Pollak 1960: 35). Seitdem werden in der Romanistik die beiden Kategorien meist getrennt. ${ }^{9}$ Der kanadische Romanist Henry Schogt sieht in der Unterscheidung von ,Aspekt' und ,Aktionsart' eine Notwendigkeit; er umschreibt dies mit folgenden Worten:

„Il ne faut [...] pas confondre cet aspect d'ordre lexical, et l'aspect $[\ldots]$ qui a un caractère purement grammatical [...] Toute classification qui confond ainsi deux domaines différents est vouée à l'échec [...]“. (Schogt 1964: 1)

Bei aller Deutlichkeit wird mit dieser Warnung allerdings nicht unterstellt, dass es 
keine Berührungen zwischen ,Aspekt' und ,Aktionsart' geben kann. Aus heuristischen Gründen erscheint die prinzipielle Trennung der lexikalischen und der grammatischen Ebene, für die Schogt plädiert, grundsätzlich als sinnvoll und angebracht.

Was die Literaturlage in der Romanistik angeht, so ist die Zahl der Veröffentlichungen zum Thema ,Aspekt' zwar beträchtlich, aber durchaus nicht unüberschaubar. In den letzten Jahren sind nur noch wenige einschlägige Publikationen vorgelegt worden. ${ }^{10}$ Eine der letzten - sie stammt von der Regensburger Romanistin Julia Mitko - beginnt mit der resignierenden Feststellung, dass man die „Diskussion über Aspekt im Romanischen als beendet ansehen könnte“ (J. Mitko 2000: 3; die Autorin gewinnt dann allerdings doch noch einige interessante neue Einsichten in die Problematik). Die Aktionsarten im Romanischen wurden im Gegensatz zu den Aspekten bisher ausgesprochen stiefmütterlich behandelt; einschlägige Monographien sind mir nicht bekannt geworden. Ich will deshalb den Aktionsarten in meiner Darstellung mehr Raum geben als den Aspektoppositionen.

2.

Zunächst aber zur Problematik der Aspekte im Romanischen. Der Terminus ,Aspekt' stammt, wie allgemein bekannt ist, aus der Slawistik. ${ }^{11}$ Er benennt die in den slawischen Sprachen gegebene Möglichkeit, mit morphologischen Mitteln „jedes Verb auf jeder Zeitstufe mit zwei morphologisch differenzierten Formen zu markieren“ (Klein 1974: 77). Es bedarf keiner weiteren Erläuterung, dass im Romanischen derartige Aspektoppositionen nicht gegeben sind. ${ }^{12}$ Ulrike Schwall unterscheidet deshalb zwischen ,Aspektualität' im allgemeinen und ,Aspekten' im besonderen, wobei sie den letztgenannten Terminus für morphologisch markierte Erscheinungen, wie sie in den slawischen Sprachen gegeben wird, reserviert. ${ }^{13}$ Weniger weitreichend ist eine von Georges Mounin vorgeschlagene, modifizierte Definition des Terminus ,Aspekt', die auch auf die romanischen Sprachen Anwendung finden kann. Mounin bestimmt den ,Aspekt' als eine

„[...] catégorie grammaticale [...] qui manifeste le point de vue sous lequel le locuteur envisage l'action exprimée par le verbe" (Mounin 1974, unter aspect).

Der französische Linguist betont also den funktionalen Gesichtspunkt, nach dem der Aspekt Ausdruck eines subjektiven „Blickwinkels“ des Betrachters auf das bezeichnete Geschehen ist. ${ }^{14}$ Eine besondere morphologische Ausprägung dagegen (wie im Slawischen) müssen die Aspektoppositionen Mounins Definition zufolge nicht haben - durch die Definition wird allerdings auch nicht ausgeschlossen, dass eine solche vorhanden sein kann.

Die wichtigste Aspektopposition im Romanischen ist eine solche, bei der durchaus eine formale Differenzierung beteiligt ist. Es handelt sich um einen Gegensatz im Bereich der Vergangenheitstempora. ${ }^{15}$ Im Französischen, auf das ich hier besonders Bezug nehmen will, sind dies das ,imparfait' einerseits, das ,passé composé' bzw. das (ausschließlich schriftsprachliche) ,passé simple' andererseits. Zur Veranschaulichung der Funktionen, welche den unterschiedlichen Vergangenheitstempora zukommen, 
können die folgenden Sätze dienen (ich füge dem französischen Beispielsatz einen spanischen und einen italienischen Satz bei, um die Vergleichbarkeit der Phänomene in den unterschiedlichen romanischen Sprachen zu verdeutlichen): ${ }^{16}$

1a) Quand je sortais de la maison, j'ai rencontré un ami

1b) Cuando salía de la casa, encontré a un conocido

1c) Quando usciva della casa, incontrai un amico

Das Verdienst, den Parallelismus zwischen diesem Teil der romanischen Verbgrammatik und dem russischen Aspektsystem herausgearbeitet zu haben, kommt dem bereits erwähnten Wolfgang Pollak zu. Dieser Romanist stellte schon im Jahre 1960 fest, dass das französische ,imparfait' funktional dem imperfektiven Aspekt des russischen Verbs im Vergangenheitstempus entspricht, während das ,passé simple' und das ,passé composé' mit dessen perfektivem Aspekt verglichen werden können. ${ }^{17}$ Wolfgang Pollak bezeichnet Satzfolgen des oben wiedergegebenen Typs als ,Inzidenzschemata'; er sieht in der „oppositiven Konstellation im Sinne des Inzidenzschemas“ geradezu ein konstitutives Element der Aspektopposition, die in den Vergangenheitstempora des Französischen gegeben ist (W. Pollak 1960: 40 bzw. 204). Im Konzept des Inzidenzschemas, das für Pollaks Ansatz charakteristisch ist, ist eine mögliche Überschreitung der Satzgrenze bereits angelegt. Der von Harald Weinrich vorgebrachte Vorwurf, es handle sich bei der ganzen Theorie um einen Rückfall in die „bequeme Satzsyntax“, ist (vgl. Klein 1974: 81) nicht gerechtfertigt.

Nach Horst G. Klein, der Pollaks Argumentation aufnimmt, liefert das im ,imparfait' verwendete Verb „in seiner Prozessualität gewissermaßen die Handlungs- und Geschehensbasis für eine perfektiv (Passé composé oder simple) gekennzeichnete Handlung [...]". ${ }^{18}$ Klein sieht mit Pollak in der aspektuellen Opposition der beiden Tempora drei Momente gegeben (Klein 1974: 80f.; vgl. auch Schwall 1991: 105):

„Die Aspektdualität ist

morphologisch charakterisiert, indem jedes Verb des Französischen auf der Vergangenheitsstufe zwei morphologisch differenzierte Formen besitzt, die zueinander in Opposition stehen,

b) semantisch differenziert, indem diese beiden Formen bewirken, dass der Handlungsablauf verschieden anvisiert wird, ohne jedoch zeitlich zu differenzieren,

c) syntaktisch determiniert dadurch, dass es strukturtypologische ,patterns' gibt, in denen sich die Aspektopposition konstituiert."

Pollaks Erklärung des Unterschiedes von ,imparfait' und ,passé simple' als aspektuelle Opposition ist in der Romanistik bis heute nicht widerlegt worden. Der bereits erwähnte Harald Weinrich hat in seinem berühmten Buch Tempus. Besprochene und erzählte Welt (Weinrich 1977) Pollaks Standpunkt kritisiert und versucht, die Anwendbarkeit der Aspekttheorie auf die romanischen Sprachen generell in Frage zu stellen. ${ }^{19}$ Seine Kritik, die von vereinzelten Sonderfällen der Tempusverwendung ausging und generell eher auf literarischen Kriterien der Textinterpretation als auf linguistischer Argumentation 
beruhte, vermochte die Mehrzahl der Linguisten allerdings nicht zu überzeugen. ${ }^{20}$ Mit Pollaks Erklärung kompatibel ist hingegen eine Theorie, die wenige Jahre nach den Studien zum Verbalaspekt von dem Heidelberger Romanisten Klaus Heger präsentiert wurde. Nach Heger erfolgt bei Verwendung des ,imparfait' die Darstellung „von innen [...], d.h. von einem sich innerhalb seines Ablaufs befindenden Bezugspunkt her." (Klein 1974: 27) Dagegen wird beim Gebrauch von ,passé composé' und ,passé simple' das jeweils bezeichnete Geschehen sozusagen „von außen“ betrachtet. Diese Erklärung ist mit Pollaks Theorie von Zusammenwirken von Hintergrund und Handlung nicht nur vereinbar; sie ist im Grunde in dieser enthalten. Auch Julia Mitko, welche die bisher aktuellste Arbeit zum Thema vorgelegt hat, macht sich diesen Standpunkt letztendlich zu eigen. Sie charakterisiert sowohl das slawische als auch das romanische Aspektsystem damit, dass in beiden Sprachenfamilien die gegebene grammatikalische Differenzierung „,...] erlaubt, ein Geschehen von außen in Globalität oder von innen in Partialität zu betrachten.“ (Mitko 2000: 3) Die Gegenüberstellung von „Innen-“ und „Außenperspektive“ bietet gegenüber der inhaltlich sehr ähnlichen Erklärung Pollaks den Vorteil, dass sie anschaulicher und terminologisch einfacher ist.

In jüngster Zeit ist verschiedentlich versucht worden, Pollaks Ansatz weiterzuentwickeln, ohne den Kern seiner Argumentation in Frage zu stellen. So bringt z.B. die erwähnte Julia Mitko die Theorie des Wiener Romanisten in Verbindung mit der These, dass Situationstypen Affinitäten zu gewissen Aspekten aufweisen können (im Französischen also auch zu bestimmten Tempora der Vergangenheit; Mitko 2000: 81; ausführliche Darstellung ebd., pp. 109-177). Die Regensburger Autorin weist auch darauf hin, dass bestimmte Phasenverben nicht mit Tempora, die den perfektiven Aspekt ausdrücken (im Französischen also ,passé composé' oder ,passé simple') kombiniert werden können (vgl. Mitko 2000: 180). So ist jeweils der zweite Satz der folgenden Beispielpaare ungrammatisch, weil die Phasenverben aller (faire qc) und venir de (faire qc) nicht mit dem ,passé simple' kompatibel sind:
2a) J'allais sortir de la maison
2b) *J'allai sortir de la maison
3a) Je venais de rencontrer un ami
3b) *Je vins de rencontrer un ami

Die Suche nach aspektuellen Strukturen blieb im Romanischen - anders als im Slawischen - lange Zeit auf die Tempora der Vergangenheit beschränkt. ${ }^{21}$ Erst in allerjüngster Zeit wurden auch andere Tempora in die aspektorientierte Betrachtung einbezogen. Besonders bemerkenswert ist in diesem Zusammenhang der Versuch der beiden Nachwuchsromanistinnen Angela Schrott und Monika Sokol, die Tempora der Zukunft aus dieser Perspektive zu untersuchen. Ebenso wie für den Ausdruck des Vergangenen verfügt das Französische ja auch für die Bezeichnung des Zukünftigen über mehrere, unterschiedliche Möglichkeiten. Diese sind das ,futur simple' (je chanterai), das ,futur périphrastique' (je vais chanter) und das ,présent futural' (je chante [maintenant)). Die traditionelle Erklärung läuft darauf hinaus, die Entscheidung des Sprechers für das eine oder das andere Tempus mit dem größeren oder geringeren zeitlichen Abstand des zukünftigen Geschehens von der Gegenwart des Sprechers zu begründen. Je vais chanter bezieht sich demnach auf ein Geschehen, welches unmittelbar bevorsteht (vgl. 
z.B. die Regel der Grammaire Larousse: „la périphrase marque un futur lié à l'instant présent“, Chevalier et al. 1964: 352, vgl. auch Grevisse 1986: 1230), je chanterai dagegen auf ein solches in fernerer Zukunft. Diese funktionelle Differenzierung lässt sich allerdings an der sprachlichen Realität nur sehr eingeschränkt belegen. ${ }^{22}$ Schrott und Sokol bringen nun - teilweise unter Rückgriff auf Vorarbeiten von Schogt, Coseriu, Wilmet und Wunderli - den in diesem Zusammenhang neuen Gesichtspunkt der Aspektdifferenzierung ins Spiel. Angela Schrott zufolge sind die folgenden Beispielsätze nicht synonym (Schrott 1997: 107, Hervorhebungen im Orig.):

4a) Il se jettera en bas de la falaise

4b) Il va se jeter en bas de la falaise

Den semantischen Unterschied, der zwischen den beiden Sätzen besteht, beschreibt Schrott wie folgt:

„Während beim futur simple die Vorhersage nur dann zutrifft, wenn der Vorsatz in die Tat umgesetzt wird, ist die Aussage im futur périphrastique auch dann wahr, wenn nur der Wille zur Tat vorhanden ist, diese aber nicht ausgeführt wird." (Schrott 1997: 107)

Diese Interpretation impliziert, dass bei dem ersten der hier behandelten Sätze der Prozess des Sich-Hinab-Stürzens sozusagen aus der Außenperspektive dargestellt wird, während bei dem zweiten die Betrachtung „von innen heraus“ erfolgt. Eine solch „imminente“ Perspektive ist beim ,futur périphrastique' häufig gegeben, wie die folgenden Beispielsätze zeigen: ${ }^{23}$

5a) [Sabines, (Les), célèbre tableau de David, (Louvre),] Romulus va lancer son javelot sur Tatius qui, à demi incliné, attend le coup pour le parer

5b) Je sens que je vais vous détester

Bei der Übersetzung ins Deutsche ist anstelle des Futurs eine periphrastische Formulierung zu verwenden, die wiederum der Tatsache Rechnung trägt, dass das bezeichnete Geschehen aus der Innenperspektive betrachtet wird:24

6a) [...] Romulus ist dabei, seinen Speer auf Tatius zu werfen, der halbgeneigt den Schlag erwartet, um ihn abzuwehren

6b) Ich fühle, wie ich anfange, Sie abscheulich zu finden

Mit dem Ansatz Angela Schrotts vergleichbar ist die Position Monika Sokols. Sie sieht bei den folgenden französischen Sätzen ebenfalls keine Bedeutungsgleichheit gegeben, was aus den vorgeschlagenen deutschen Übersetzungen deutlich wird:

7a) À huit heures, il finira son travail

7b) À huit heures, il va finir son travail

8a) Um acht Uhr wird er mit seiner Arbeit fertig sein

8b) Um acht Uhr wird er loslegen, um seine Arbeit zu Ende zu bringen (Sokol 1999: 150)

Im ersten Satz drückt Sokol zufolge das ,futur simple' den perfektiven Aspekt aus, während im zweiten das ,futur périphrastique' den imperfektiven Aspekt wiedergibt. ${ }^{25}$ Verwendungen des zweiten Typs sind, wie Sokol ausführt, derzeit noch nicht die Regel; die Konstruktionen aus einer Form , aller + Infinitiv' sind ihrer Ansicht nach allerdings dabei, sich hin zu imperfektiven Ausdrücken zu entwickeln (vgl. Mitko 2000: 13). Das 
,présent futural' schließlich ist nach Sokol im Rückgang begriffen; es wird zunehmend durch das ,futur périphrastique' ersetzt. Somit zeichnet sich, wenn Monika Sokol recht hat, eine „komplementäre Verteilung der Funktionsbereiche von ,futur simple' und ,futur périphrastique' ab. ${ }^{26}$ Maßgeblich für die Abgrenzung der beiden Formen ist hierbei das Vorliegen des aspektuellen Moments der Außen- bzw. Innenperspektive.

Ein Phänomen, das immer wieder mit der aspektuellen Differenzierung in ,Innenund ,Außenperspektive' in Verbindung gebracht wurde, ist schließlich das der französischen ,Verlaufsform' être en train de faire qc und ihrer Entsprechungen in den übrigen romanischen Sprachen. Die Romanistik hat sich bei der Beschreibung dieser Erscheinung bisher weitgehend am Vorbild der englischen ,progressive form' orientiert. Ähnlich wie bei der englischen Form wird auch bei der französischen das im Verb geschilderte Geschehen aus der „Innenperspektive“ betrachtet. Trotz des Vorliegens offensichtlicher Übereinstimmungen darf aber nicht übersehen werden, dass die französische ,Verlaufsform' nur bedingt mit der englischen vergleichbar ist. Zum einen weist sie - anders als die englische - starke Kombinationsrestriktionen auf; im allgemeinen wird sie nur in Verbindung mit belebten Subjekten gebraucht (vgl. Mitko 2000: 185). Zum anderen liegt die Verwendung oder Nichtverwendung von être en train de faire qc immer noch weitestgehend im Ermessens des Sprechers; die Form ist generell weniger stark grammatikalisiert als die englische ,progressive form'. Die französische ,Verlaufsform' unterscheidet sich in dieser Hinsicht auch von der (ansonsten funktional ähnlichen) spanischen Gerundialkonstruktion estar haciendo (alguna cosa). So ist, wie Juan Diego Quesada feststellt, die Verwendung des Gerundiums im Spanischen in bestimmten Fällen obligatorisch, während dies für die französische ,Verlaufsform' nicht gilt (Quesada 1994: 220, nur spanische Beispiele):

9a) ¿Qué haces? ¿Qué estás haciendo?

9b) ${ }^{*}$ Como. Estoy comiendo

10a) Qu'est-ce que tu fais? Qu'est-ce que tu es en train de faire?

$10 \mathrm{~b})$ Je mange. Je suis en train de manger

Eine weitere Besonderheit des Spanischen gegenüber dem Französischen besteht darin, dass die erstgenannte Sprache über ein regelrechtes System von Gerundialformen verfügt. Neben der hier behandelten Form estoy haciendo existieren im Spanischen auch ando haciendo, voy haciendo, vengo haciendo und sigo haciendo. Der unlängst verstorbene Eugenio Coseriu fasst diese unterschiedlichen Formen in dem (unübersetzbaren) Terminus „Schau“ zusammen (vgl. Coseriu 1976: 99ff.). Er unterscheidet im einzelnen „Winkelschau“, „komitative Schau“, „prospektive“, „restrospektive“ und „kontinuative“ Schau. Coseriu sieht in all diesen Konstruktionen den Ausdruck einer "partikularisierenden“ Sichtweise des Betrachters auf das bezeichnete Geschehen. Diesen stellt er die "globale“ Schau gegenüber, die in Konstruktionen wie tomo y me voy (schwer übersetzbar, etwa „ich entschließe mich zu gehen”, s. Coseriu 1966: 42, vgl. auch Coseriu 1976: 102f., oder "jetzt gehe ich aber") realisiert wird. Coserius Schüler Wolf Dietrich hat versucht, die Herkunft dieser "periphrastischen Verbalaspekte“ der Kategorie „Schau“ auf den Einfluss zurückzuführen, den das Griechische in der Spätantike auf das Latein ausgeübt hat. ${ }^{27}$ Die Fachwelt hat diese These sehr unterschiedlich aufgenommen;28 wir können dem hier nicht weiter nachgehen. 
3.

Ich komme zu den Aktionsarten. Die Aktionsarten werden heute meist - ganz im Sinne Sigurd Agrells - als „objektiv in der Verbbedeutung enthaltene Spezifizierung[en] des Verbalvorgangs" (Bußmann 1990, unter Aktionsart) definiert, als inhaltliche Charakteristika, die „dem einzelnen Verbum inhärent [...] sind“. ${ }^{29}$ Es handelt sich bei den Aktionsarten also um semantische Merkmale von weitreichender Gültigkeit. Dem Sprecher steht es im Hinblick auf die Aktionsarten - anders als in Bezug auf die Aspekte - nicht frei, bei ein und dem selben Verb zwischen verschiedenen Möglichkeiten auszuwählen. Natürlich können die semantischen Momente, die in den Aktionsarten realisiert sind, auf der Ebene der Sätze und Texte auch auf andere Weise ausgedrückt werden (etwa durch Periphrasen). Vereinzelt ist deshalb vorgeschlagen worden, analog zur Trennung von ,Aspekten' und ,Aspektualität' auch zwischen ,Aktionsarten' und ,Aktionalität' zu unterscheiden (vgl. Czarnecki 2000: 11).

Bei der Definition der ,Aktionsarten' wird häufig auch auf die Notwendigkeit von deren morphologischer Realisierung verwiesen. So ist nach Renate Steinitz grundsätzlich „[...] das Vorhandensein einer grammatischen Beziehung zwischen einfachem und abgeleitetem Verb, die mit der semantischen Beziehung [...] korreliert, konstitutiv für die Annahme einer Kategorie ,Aktionsart' im System einer Sprache" (Steinitz 1981: 41). Als Beispiele können Wortreihen wie dt. blühen/aufblühen/erblühen/verblühen (vgl. Gipper 1972: 187f.) oder auch (semantisch ganz anders einzustufende) Wortpaare wie fahren/ abfahren, denken/ausdenken dienen. Das Vorliegen morphologischer Unterscheidungsmöglichkeiten wird jedoch nicht von allen Linguisten als Bedingung für die Annahme von Aktionsarten angesehen; in Grammatiken des Deutschen werden vielfach auch nicht abgeleiteten Verben wie schneien, regnen, arbeiten oder sterben bestimmte Aktionsarten zugewiesen. ${ }^{30}$ Auch in der oben zitierten, klassischen Definition Agrells wird die Kategorie der Aktionsarten sowohl auf Simplizia als auch auf Derivatformen angewandt.

Was Zahl und Klassifikation der Aktionsarten angeht, so besteht weitgehende Übereinstimmung darin, dass zwar bestimmte Parallelen zu den Aspekten bestehen, dass aber die Einteilung der Aktionsarten jener der Aspekte nicht völlig entspricht. Eine der ausführlichsten Listen findet sich im Handbuch der Linguistik (Stammerjohann et al. 1975). Die Autoren dieses Werks unterscheiden nicht weniger als acht Aktionsarten. Sie nennen die ,faktitive' Aktionsart (dt. röten, töten), die hiermit eng verwandte ,kausative' (verschwenden im Gegensatz zu verschwinden), die ,iterative' (dt. stechen/sticheln oder auch lat. dico/dicto), die ,intensive' (schnitzen zu schneiden), ,ornative' (verzieren zu zieren), ,privative' (entmachten; hier fehlt allerdings das Simplex), ,inchoative' (dt. erblühen zu blühen oder lat. ardesco zu ardo) und ,resultative' (zerkrümeln zu krümeln, aufessen zu essen usw.). ${ }^{31}$ Außerdem verweisen sie auf die Opposition der Aktionsarten ,durativ' und ,punktuell', die dem aspektuellen Gegensatz, imperfektiv'/,perfektiv' in gewisser Weise vergleichbar ist. Andere Aufzählungen nennen nur wenige unterschiedliche Aktionsarten. Praktisch immer genannt werden die Kategorien ,durativ' und ,punktuell', weniger regelmäßig, jedoch immer noch häufig, die Aktionsart, iterativ'. Die verwendete Terminologie ist in der einschlägigen Literatur alles andere als einheitlich. Unklar ist 
vor allem das Verhältnis der Dichotomie ,durativ'/,punktuell' zu dem aspektuellen Gegensatz , perfektiv'/,imperfektiv' ${ }^{32}$

Die Aktionsarten im Romanischen sind, wie ich eingangs schon erwähnte, bisher nur in Ansätzen untersucht worden. Dies mag damit zusammenhängen, dass die morphologische Markierung der Aktionsarten in den romanischen Sprachen weniger deutlich in Erscheinung tritt als etwa im Deutschen (oder auch noch im Lateinischen). Zwar lassen sich im Französischen, um das es hier gehen soll, den Simplexformen der Verben nicht selten abgeleitete Formen zuordnen. Beispiele sind etwa craqueler (frequentativ-diminutive Aktionsart zu craquer), mordiller (frequentative Aktionsart zu mordre) oder siffloter (iterativ-frequentative Aktionsart zu siffler). ${ }^{33}$ Aus den Beispielen lässt sich bereits erahnen, dass die Zahl der morphologisch realisierten Kategorien im heutigen Französisch eng begrenzt ist. Nach Ulrich Wandruszka finden im Grunde nur die Aktionsarten ,augmentativ', , frequentativ' und ,diminutiv' in größerem Umfang auf der Ebene der Wortbildung ihren Ausdruck. ${ }^{34}$ Wenn in der Fachliteratur von den ,caractères de l'action' im Französischen die Rede ist, so sind damit ganz überwiegend semantische Merkmale ohne morphologische Realisierung gemeint. ${ }^{35}$ Dies schließt nicht aus, dass die inhaltlichen Gegebenheiten auf einer anders gelagerten formalen Ebene (etwa im kombinatorischen Verhalten der betroffenen Verben) sichtbar werden.

Die Diskussion in der Romanistik wird z.T. dadurch verkompliziert, dass unter die „Aktionsarten“ teilweise auch periphrastische Konstruktionen gerechnet werden. So rechnet z.B. Otto Ducháček zu den „terminativen“ (d.h. resultativen) Verben einerseits (nicht abgeleitete) Wörter wie arriver und atteindre, deren Signifikate ein entsprechendes Inhaltsmerkmal aufweisen. Andererseits verweist er in diesem Zusammenhang aber auch auf Syntagmata wie chanter jusqu'à la fin, die nur als Ganzes terminativen Charakter besitzen und bei denen von einer Verbinhärenz der terminativen Aktionsart keine Rede sein kann (Ducháček 1966: 172f.). Die Vermengung der beiden Erscheinungen hat beträchtliche Verwirrung gestiftet und die Erforschung der Aktionsarten im Romanischen erheblich erschwert. ${ }^{36}$ In der folgenden Darstellung soll der Bereich der Periphrastik, dessen Semantik mit der oben formulierten Definition der Aktionsarten als verbinhärente Merkmale nicht kompatibel ist, ausgeklammert bleiben.

Die Aktionsarten, die in der Romanistik am häufigsten erwähnt werden, sind - ähnlich wie in der Germanistik und anderswo - die Kategorien ,punktuell' und ,durativ' ${ }^{37}$ Neben diesen finden auch Ausdrücke wie frz. ,momentané', ,complétif', ,conclusif', ,terminatif', ,résultatif' auf der einen, ,progressif' und intensif' auf der anderen Seite Verwendung (all diese Termini wurden von Otto Ducháček vorgeschlagen, vgl. Ducháček 1966: 166). Bei anderen Autoren ist zuweilen auch von ,imperfektiver' und ,perfektiver' Aktionsart die Rede. Es ist durchaus unklar, ob mit den divergierenden Termini unterschiedliche Dinge benannt werden sollen oder ob es sich um Synonyme handelt. Das Problem soll mit Bezug auf das Französische auf den folgenden Seiten diskutiert werden. Als sprachliches Bezugsmaterial dienen bestimmte Verben der visuellen Wahrnehmung, speziell die Verben voir und regarder. ${ }^{38}$

Die beiden Verben voir und regarder sind die mit Abstand am häufigsten vorkommenden Wahrnehmungsverben des Französischen. ${ }^{39}$ Sie entsprechen semantisch ungefähr (nicht 
vollständig) den deutschen Verben sehen und ansehen/betrachten. Was den semantischen Unterschied zwischen den beiden französischen Wörtern angeht, so ist ein Rückgriff auf die Aktionsarten ,punktuell' und ,durativ' z.T. durchaus hilfreich.

Vergleichsweise einfach zu beschreiben ist die Semantik des Verbs voir. Das Wort verfügt offensichtlich über ein Inhaltsmerkmal ,punktuell'. Als Beleg können die folgenden Beispielsätze dienen, in denen das finite Verb voir als Objektergänzung eine Infinitivkonstruktion regiert:

11a) En voyant apparaître Boris, les soldats rirent et crièrent ... (Sartre, La mort dans l'âme, p. 67)

11b) Chick le vit, étonné d'abord, devenir brusquement de la couleur de la glace (Vian, L'écume des jours, p. 84)

Die Infinitivkonstruktion benennt hier ein plötzliches Erscheinen einer Person im Gesichtskreis des wahrnehmenden Agens, also ein momentanes, punktuelles Geschehen. Voir kann auf solche Prozesse referieren, regarder dagegen nicht (in den obenstehenden Beispielsätzen wäre eine Substitution von voir durch regarder nicht zulässig).

Anders als regarder kann voir auch explizite Prozesse des Konstatierens von Sachverhalten denotieren, die per definitionem punktueller Art sind. Diese liegen vor allem in solchen syntaktischen Konstruktionen vor, in denen das finite Verb einen durch die Konjunktion que eingeleiteten Objektsatz regiert. In den folgenden Sätzen ist voir wiederum nicht durch regarder ersetzbar:

12a) L'autodidacte, qui voit que j'écris, m'observe avec une concupiscence respectueuse (Sartre, La nausée, p. 49)

12b) ... quand elle se retourna, il vit que son visage était couvert de larmes (Camus, La peste, p. 18)

12c) De la pente qui descendait vers la mer nous avons vu qu'il y avait déjà quelques baigneurs (Camus, L'étranger, p. 80f.)

Das konstative Moment kommt auch in solchen Konstruktionen zum Ausdruck, in denen das Verb ein komplexes Objekt regiert, das aus einem Nomen/einer Nominalphrase und einem Objektattribut besteht. Voir kann, wie die folgenden Beispielsätze zeigen, in solchen Verwendungen vorkommen. Für regarder gilt dies dagegen nicht:

13a) Cette ville où le sang ruisselait, il n'avait cessé de la voir calme et amie ... (Malraux, L'espoir, p. 116)

13b) Lombard est drôle, dit Anne. Je l'ai vu très amusant (Sagan, Bonjour tristesse, p. 24)

13c) Il était ému de la voir si mal habillée (Simenon, Les suicidés, p. 36)

Voir kann somit als punktuelles, genauer gesagt als konstatives Verb eingestuft werden.

Die Bedeutung von regarder ist demgegenüber weniger leicht zu ermitteln. Das Wort weist, wie die durchgeführten Austauschproben zeigen, eindeutig nicht die ,punktuelle' Aktionsart auf. Es ist jedoch problematisch, hieraus auf das Vorhandensein eines Merkmals ,durativ' zu schließen, wie dies z.B. Eugenio Coseriu tut. ${ }^{40}$ Regarder kommt zwar häufig in Kombinationen mit solchen Infinitivkonstruktionen vor, die ein länger andauerndes Geschehen bezeichnen. ${ }^{41}$ Ebenso kann das Wort aber auch zur 
Bezeichnung kurz andauernder Vorgänge dienen, was beim Vorliegen eines Merkmals ,durativ' nicht möglich wäre. Beispielsatz:

14) Elle se regardait furtivement dans un miroir pour s'assurer qu'elle était correcte (Simenon, Les suicidés, p. 81)

Was regarder semantisch kennzeichnet, ist ein anderes Moment, nämlich das des Engagement des Agens. Das Verb benennt eine willentliche Handlung („handling“ im Gegensatz zur bloßen „aktivitet“ nach Per Durst-Andersen und Karl-Henrik Lund ${ }^{42}$ ), die auf das visuelle Erkennen von Sachverhalten abzielt. Diese Handlung ist oft von einer gewissen zeitlichen Dauer (daher wohl Coserius Einstufung als ,duratives' Verb). Dies muss aber nicht zwangsläufig der Fall sein. Ich möchte das Moment des Engagements mit den folgenden Beispielsätzen veranschaulichen, in denen der Infinitiv regarder auf ein finites Verb folgt, welches explizit ein bestimmtes Engagement des Agens bezeichnet:

15a) Ils sont trois ou cinq, je ne sais pas, je n'ai pas le courage de les regarder (Sartre, La nausée, p. 35)

15b) Quand je me forçai à regarder, le point noir avait disparu (Camus, La chute, p. 114)

15c) Par pudeur, je n'osai pas les regarder, puis leur silence me força à lever les yeux (Sagan, Bonjour tristesse, p. 59)

Der Handlungscharakter des Verbs regarder wird dadurch unterstrichen, dass das Wort vorzugsweise mit solchen adverbialen Bestimmungen kombiniert wird, die ein Engagement oder eine entsprechende psychische Verfasstheit des Agens umschreiben. Auch hier nenne ich einige einschlägige Beispielsätze:

16a) Lola le regarda attentivement (Sartre, La mort dans l'âme, p. 226)

16b) Pendant ses effusions, elle restait les yeux ouverts, à le regarder curieusement ... (Simenon, Les suicidés, p. 135)

16c) Il s'est tourné vers Brunet et le regarde haineusement (Sartre, La mort dans l'âme, p. 226)

Bei dem oben beschriebenen Verb voir ist die Aktionsart ,Handlung' offensichtlich nicht gegeben. Dies zeigt sich unter anderem darin, dass dieses Wort in keinem der soeben aufgezeigten Kontexte anstelle von regarder verwendet werden kann. Das Wort bezeichnet statt dessen das Ergebnis der durch regarder ausgedrückten Handlung, eben das visuelle Erkennen. Dies wird unter anderem dadurch deutlich, dass voir mit solchen Adverbien kombiniert werden kann, die - anders bei regarder - die Qualität des eigentlichen Konstatierens der Sachverhalte benennen. Beispielsätze:

17a) Scali voyait distinctement les points blancs des turbans maures ... (Malraux, L'espoir, p. 105)

17b) On voyait nettement dans l'oreiller le creux qu'avait sculpté la tête de Bachelin (Simenon, Les suicidés, p. 137)

17c) On voyait clairement que le printemps s'était exténué ... (Camus, La peste, p. 107)

Regarder benennt demzufolge eine willentlich gesteuerte Aktivität, für die ich den Terminus ,Handlung' vorgeschlagen habe, voir deren Ergebnis. Die beiden Verben bezeichnen grundsätzlich unterschiedliche Prozesse. Das Moment ,Handlung' darf deshalb nicht mit der Aktionsart ,imperfektiv', die im klassischen Inventar der Aktionsarten vorkommt, gleichgesetzt werden. Der Bezug der beiden Verben 
aufeinander kommt zuweilen auch in den Texten explizit zum Ausdruck. Beispielsätze:

18a) La maîtresse a regardé pour voir si la classe et nous étions bien propres ... (Sempé, Le petit Nicolas, p. 39)

18b) Après notre conversation, ..., je l'ai regardé et j'ai vu que c'était un homme aux traits fins ... (Camus, L'étranger, p. 100)

18c) Mathieu regarda son bracelet-montre et vit qu'il était six heures (Sartre, La mort dans l'âme, p. 230)

Besonders deutlich wird die semantische Unterschiedlichkeit der beiden Verben dadurch, dass in den Texten zuweilen ausdrücklich klargestellt wird, dass voir und regarder unterschiedliche Prozesse bezeichnen. Die durch regarder bezeichnete Handlung muss nicht unbedingt zu dem Ergebnis führen, das durch voir benannt wird. Beispiele:

19a) Combien d'heures restera-t-il ainsi, regardant sans la voir une étroite fenêtre grillée ...? (Bernanos, Sous le soleil de Satan, p. 271)

19b) Au fond d'un taxi qui roulait dans les rue vides, M. Grandvalet regardait sans le voir le dos du chauffeur ... (Simenon, Les suicidés, p. 152)

Die explizite Trennung der bezeichneten Prozesse in den Texten stellt allerdings eher die Ausnahme dar. Die mit regarder benannte Handlung führt meistens eben doch zu dem Ergebnis, das durch voir ausgedrückt wird. Folglich kommen auch solche Sätze vor, in denen die beiden Verben problemlos gegeneinander ausgetauscht werden können. Es handelt sich in diesen Fällen, in strukturalistischer Terminologie ausgedrückt, um Neutralisierungen. Beispielsätze:

20a) Dis-y seulement que j'emmène la petite au Mont-de-piété pour voir le spectacle (Queneau, Zazie dans le métro, p. 138)

20b) Es-tu allé voir le crèche de Noël, au moins? (Simenon, Les demoiselles de Concarneau, p. 70)

20c) Voyez-le, cet ange de la peste, beau comme Lucifer et brillant comme le mal lui-même ... (Camus, La peste, p. 115)

Die beschriebene Opposition der Verben regarder und voir stellt im französischen Wortschatz keine isolierte Erscheinung dar. Auch zahlreiche andere Verben weisen die Aktionsarten ,Handlung' und ,Ergebnis' auf und stehen in ähnlicher Weise in Gegensatz zueinander wie die beiden beschriebenen Wörter der visuellen Wahrnehmung.

Weitgehend vergleichbar sind beispielsweise andere Wahrnehmungsverben wie écouter „zuhören“ und entendre „hören“, ebenso flairer „wittern“/goûter „kosten“/tâter „betasten“ einerseits, sentir „riechen, schmecken, spüren“ andererseits. Auch zahlreiche Verben, die keinen Prozess der sinnlichen Wahrnehmung bezeichnen, sondern auf intellektuelle Vorgänge referieren, lassen sich den Aktionsarten ,Handlung' und ,Ergebnis' zuordnen. Ich nenne als Beispiele für die erste Gruppe die Wörter analyser, étudier, examiner, explorer, inspecter, méditer, als solche für die zweite Gruppe constater, noter und reconnaître. Ähnlich strukturiert sind letztendlich auch die Verben der sprachlichen Äußerung. Die wichtigsten Vertreter dieser Gruppe sind parler "sprechen“ (handlungsorientiert) und dire „sagen“ (ergebnisorientiert). Eine systematische Beschreibung dieser Oppositionen, die bisher erst in Ansätzen geleistet ist, kann im Idealfall zu Einblicken in eines der semantischen Grundstrukturmuster des französischen Verbalwortschatzes führen. 
4.

Ich komme zum Schluss. Morphologisch markierte Aspektoppositionen wie in den slawischen Sprachen sind im Romanischen (natürlich) nicht gegeben. Es finden sich aber in Teilen der Grammatik Gegensätze, die funktional den Aspekten des Slawischen vergleichbar sind. Dies gilt vor allem für die Vergangenheitstempora, die - etwa im Französischen - mit dem Gegensatz von ,imparfait' einerseits, ,passé simple'/,passé composé' andererseits eine Opposition von ,imperfektivem' und ,perfektivem' Aspekt aufweisen. Weniger klar erkennbar ist eine derartige Strukturierung im Bereich des Futurs und der Verlaufsformen. Anders als in verschiedenen anderen Sprachen sind die Aktionsarten im Französischen morphologisch nicht realisiert. Jedoch gilt auch hier, dass bestimmte funktionale Parallelen zu solchen Sprachen vorliegen, in denen die Aktionsarten (auch) ein Verfahren der Wortbildung darstellen. Als besonders weitreichend erwies sich der Gegensatz ,Handlung'/,Ergebnis', dessen einer Pol dem perfektiven Aspekt vergleichbar ist. Für die künftige Wortschatzarbeit eröffnet sich hier ein ergiebiges Forschungsfeld.

\section{LITERATUR}

Bußmann, Hadumod (1990), Lexikon der Sprachwissenschaft, 2. Aufl., Stuttgart: Kröner.

Chevalier, Jean-Claude et al. (1964), Grammaire Larousse du français contemporain, Paris: Librairie Larousse.

Christmann, Hans Helmut (1959), „Zum ,Aspekt’ im Romanischen. Bemerkungen zu einigen neueren Arbeiten“, Romanische Forschungen, 71 (1959), pp. 1-16.

Coseriu, Eugenio (1966), „,Tomo y me voy'. Ein Problem vergleichender europäischer Syntax“, Vox Romanica, 25 (1966), pp. 13-55.

Coseriu, Eugenio (1976), Das romanische Verbalsystem, hg. und bearb. von Hansbert Bertsch, Tübingen: TBL-Verlag Narr.

Czarnecki, Tomasz (2000), „Zur Verwendung des Terminus ,Aspektualität' in der sprachwissenschaftlichen Forschung“, in: Kọtny, Andrzej (Hg.) (2000), Aspektualität in germanischen und slawischen Sprachen, Poznañ: Wydawnictwo Naukowe UAM.

Dietrich, Wolf (1973), Der periphrastische Verbalaspekt in den romanischen Sprachen. Untersuchungen zum heutigen romanischen Verbalsystem und zum Problem der Herkunft des periphrastischen Verbalaspekts, Tübingen: Niemeyer.

Ducháček, Otto (1966), „Sur le problème de l'aspect et du caractère de l'action verbale en français“, Le Français Moderne, 34 (1966), pp. 161-184.

Eisenberg, Peter (1999), Grundriss der deutschen Grammatik, Bd. 2, Der Satz, Stuttgart/ Weimar: Metzler.

Gipper, Helmut (1972), Gibt es ein sprachliches Relativitätsprinzip?, Frankfurt/M.: S. Fischer.

Grevisse, Maurice (1986), Le bon usage. Grammaire française, Douzème édition refondue par André Goosse, Paris/Gembloux: Duculot.

Haensch, Günther, Annette Lallemand-Rietkötter (1972), Wortbildungslehre des modernen Französisch, München: Max Hueber.

Hanckel, Walter (1930), Die Aktionsarten im Französischen, Berlin: Phil. Diss. 
Heger, Klaus (1963), Die Bezeichnung temporal-deiktischer Begriffskategorien im französischen und spanischen Konjugationssystem, Tübingen: Niemeyer.

Juilland, Alphonse et al. (1970), Frequency Dictionary of French Words, The Hague/Paris: Mouton.

Klein, Franz-Josef (1981), Lexematische Untersuchungen zum französischen Verbalwortschatz im Sinnbezirk von Wahrnehmung und Einschätzung, Genève: Librairie Droz.

Klein, Horst G. (1974), Tempus - Aspekt - Aktionsart, Tübingen: Niemeyer.

Knobloch, Johann (1961ff.), Sprachwissenschaftliches Wörterbuch., Vol. 1ff., Heidelberg: C. Winter.

Leisi, Ernst (1973), Praxis der englischen Semantik. Unter Mitwirkung von Dorothea Wagner und Walter Naef, Heidelberg: Winter.

Lund, Karl-Henrik (2003), „Aspekt, tempus og Kærlighedens ø - om narrativ progresseion i dansk og russisk“, i dette bind.

Meier-Brügger, Michael (2000), Indogermanische Sprachwissenschaft., 7., völlig neubearbeitete Aufl. der früheren Darstellung von Hans Krahe. Unter Mitarbeit von Mathias Fritz und Manfred Mayrhofer, Berlin/New York: de Gruyter.

Meyer-Lübke, Wilhelm (1899), Grammatik der romanischen Sprachen, Bd. 3, Romanische Syntax, Leipzig: Fues's Verlag (R. Reisland).

Mitko, Julia (2000), Aspekt im Französischen. Eine semantisch-funktionelle Analyse, Tübingen: Narr.

Mounin, Georges (1974), Dictionnaire de la linguistique, Paris: Presses universitaires de France.

Naert, Pierre (1960), „Mode de présentation, aspect, mode d'action, détermination et transitivité“, Studia Linguistica, 14 (1960), pp. 1-14.

Pollak, Wolfgang (1960), Studien zum, Verbalaspekt' im Französischen, Wien: In Kommission bei R. M. Rohrer.

Pollak, Wolfgang (1970), „Aspekt und Aktionsart“, Linguistik und Didaktik, I (1970), pp. 40-47.

Quesada, Juan Diego (1994), Periphrastische Aktionsart im Spanischen: das Verhalten einer Kategorie der Übergangszone, Frankfurt/M. etc.: P. Lang.

Rohrer, Christian (1976), Rez. zu Dietrich, Wolf (1973), Zeitschrift für romanische Philologie, 92 (1976), pp. 168-172.

Schogt, Henry G. (1964), „L'aspect verbal en français et l'élimination du passé simple“, Word, 20 (1964), pp. 1-17.

Schrott, Angela (1997), Futurität im Französischen der Gegenwart. Semantik und Pragmatik der Tempora der Zukunft, Tübingen: Narr.

Schwall, Ulrike (1991), Aspektualität. Eine semantisch-funktionelle Kategorie, Tübingen: Narr.

Sokol, Monika (1999), Das Zusammenspiel verbalkategorialer Inhalte und die französischen Futura, Tübingen: Niemeyer.

Stammerjohann, Harro, et al. (1975), Handbuch der Linguistik. Allgemeine und angewandte Sprachwissenschaft. Unter Mitarbeit von Hildegard Janssen, zusammengestellt von Harro Stammerjohann, München: Nymphenburger Verlagshandlung. 
Steinitz, Renate (1981), Der Status der Kategorie "Aktionsart" in der Grammatik (oder: Gibt es Aktionsarten im Deutschen?), Berlin: Zentralinstitut für Sprachwissenschaft der Akademie der Wissenschaften der DDR (= Linguistische Studien, Reihe A: Arbeitsberichte 76).

Stobitzer, Heinrich (1968), Aspekt und Aktionsart im Vergleich des Französischen mit dem Deutschen, Englischen und Italienischen, Tübingen: Phil. Fak., Diss. v. 8. Aug. 1968.

Wandruszka, Ulrich (1976), Probleme der neufranzösischen Wortbildung, Tübingen: Niemeyer.

Weinrich, Harald (1977), Tempus. Besprochene und erzählte Welt, Stuttgart etc.: Kohlhammer.

Wunderli, Peter (1975), Rez. zu Dietrich, Wolf (1973), Vox Romanica, 34 (1975), pp. 226232.

\section{ANMERKUNGEN}

1 Zur Diskussion des Themas seit dem 17. Jahrhundert vgl. Pollak (1960: 30-33).

2 Zitiert nach Pollak (1960: 34). Vgl. auch Klein (1974: 104), sowie Coseriu (1976: 87).

3 Die Vermengung von Aspekten und Aktionsarten kann schon bei Leskien nachgewiesen werden; vgl. Mitko (2000: 7). Nach Klein (1974: 80) zeichnet sich die „traditionelle Literatur zum Verbalaspekt" geradezu dadurch aus, dass Aspekt und Aktionsart nicht klar getrennt werden.

$4 \quad$ Ein vernichtendes Fazit zieht Klein (1974: 76). Der Autor stellt in bezug auf den Aspektbegriff fest, dass „[...] mit kaum einem anderen Terminus in der Grammatik so viel terminologischer Unfug getrieben wurde wie mit diesem [...] Es gibt kaum ein Verbalmorphem, das, wenn die Tempuskategorisierung zur Funktionsbestimmung nicht ausreichte, nicht aspektverdächtig wurde."

5 Als Beispiel vgl. Leisi (1973: 63): „Es herrscht eine große terminologische Vielfalt, um nicht zu sagen Verwirrung. Einstweilen ist es ratsam, Aspekt und Aktionsart wie folgt zu trennen: Aktionsart ist eine semantische Kategorie, d.h. die Unterschiede der Aktionsart werden durch Wechsel des ganzen Wortes ausgedrückt [...] Hiervon abzugrenzen ist der Aspekt, er ist eine grammatische Kategorie; denn Unterschiede des Aspekts werden durch grammatische Mittel ausgedrückt".

6 Vgl. Ducháček (1966: 161ff.). Der Belgier Pierre Naert (der allerdings kein Romanist ist) spricht von den „modes d'action“; vgl. Naert (1960).

7 Hanckel (1930). Bezeichnend ist eine Aussage Hanckels, nach der Aspekt und Aktionsart letztendlich dasselbe sind (ebd., p.3, Anm. 1). Kritisch hierzu Pollak (1960: 95).

8 Pollak (1960: 39). In diesem Sinne auch Klein (1974: 103): „Aktionsarten sind [...] nicht paradigmatische Bestandteile des morphologischen Inventars, sondern lexikalische Kategorien".

9 Besonders deutlich sichtbar wird dies bei Stobitzer (1968), z.B. p. 21.

10 Hier einige frühere (z.T. oben bereits genannte) einschlägige Veröffentlichungen: Hanckel (1930), Christmann (1959), Pollak (1960), Heger (1963), Schogt (1964), Stobitzer (1968), Dietrich (1973) und Klein (1974).

11 Zur Vorgeschichte des Terminus im 19. Jahrhundert vgl. Pollak (1960: 30-34).

12 Die Slawistik steht aus eben diesem Grund der Übertragung des Aspektbegriffs auf nichtslawische Sprachen im Allgemeinen skeptisch gegenüber. Vgl. Mitko (2000: 3).

13 Zum Verhältnis der beiden Termini zueinander vgl. Schwall (1991: 2): „Aspekt kann als Kernfeld der Kategorie Aspektualität aufgefasst werden, so wie Tempus als Kernfeld der semantisch-funktionellen Kategorie Temporalität angesehen werden kann." Zur Terminologie 
vgl. ebd., p. 92: „Die Termini Aspekt und Aktionsart werden von uns ausschließlich zur Deskription von Phänomenen slawischer Sprachen verwendet.“ Ausführliche Diskussion des Terminologieproblems bei Czarnecki (2000).

14 Die „semantisch-funktionelle“ Perspektive erlaubt erst den Vergleich aspektueller Erscheinungen in unterschiedlichen Sprachen; vgl. Schwall (1991: 2).

15 Nach Mitko (2000: 12) haben sich „[...] die Aspektstudien zu den romanischen Sprachen bisher nahezu ausschließlich auf die Gegenüberstellung von Bedeutungen und Textfunktionen von IMP und PS bzw. PC konzentriert [...]".

16 Nach Klein (1974: 81), leicht modifiziert. Wie die oben angeführten Beispielsätze zeigen, sind entsprechende Konstruktionen, die aus Haupt- und Nebensatz mit unterschiedlichen Vergangenheitstempora bestehen, in allen romanischen Sprachen üblich. Sie kamen im übrigen auch bereits im Latein vor.

17 Nach Klein (1974: 80). Ansätze zu der hier beschriebenen Erklärung finden sich bereits sehr viel früher, so z.B. bei Meyer-Lübke (1899: 116). Wichtig ist auch der Erklärungsansatz des Anglisten M. Deutschbein; vgl. Christmann (1959: 4).

18 Klein (1974: 82). Der Parallelismus zwischen der Organisation der französischen Vergangenheitstempora und dem russischen Aspektsystem wird dadurch unterstrichen, dass in Übersetzungen französischer Erzähltexte ins Russische das imparfait gewöhnlich durch den imperfektiven Aspekt wiedergegeben wird, während der perfektive Aspekt an die Stelle von passé composé und passé simple tritt; vgl. Mitko (2000: 14). Hiermit stimmig ist, dass „russischsprachige Grammatiken [...] nahezu ausnahmslos die französische Dichotomie Imparfait/Passé composé (bzw. simple) mit Hilfe des russischen Aspektsystems“ erklären; vgl. Klein (1974: 84).

19 Vgl. z.B. Weinrich (1977: 121f.). Die Radikalität von Weinrichs Ansatz zeigt sich u.a. darin, dass er sogar leugnet, dass die Tempora etwas mit „Zeit“ zu tun haben; siehe hierzu Klein (1974: 10), sowie Mitko (2000: 3).

20 Aufschlussreich sind in diesem Zusammenhang Weinrichs Ausführungen zum ,imparfait de rupture'; Weinrich (1977: 108f.). Zu Weinrichs methodischem Ansatz vgl. Klein (1974: 26).

21 Pollak (1960: 204f.) scheint nur in diesem Bereich überhaupt aspektuelle Strukturen anzunehmen.

22 Die Erklärung ist auch schwer mit der Tatsache vereinbar, dass die gesprochene und die geschriebene Sprache eindeutige Präferenzen zugunsten des einen bzw. des anderen Tempus erkennen lassen. Es ist kaum plausibel, dass sich die Verwendung des Futurs im „langage parlé“ vor allem auf Geschehnisse in naher, im „langage écrit“ dagegen solche in ferner Zukunft bezieht.

23 Schrott (1997: 108 bzw. 109). Der erste der beiden Sätze stammt aus einer Bildbeschreibung in einem Kunstführer.

24 Schrott (1997: 109, zweiter Satz); der erste Satz wird von Schrott nicht übersetzt.

25 Genauer hierzu Mitko (2000: 12): „Mit dem futur simple [...] liegt nach Sokol ein Marker perfektiven Aspekts vor, der nur im Zusammenspiel mit bestimmten (dynamischen und begrenzten) Situationstypen temporal-futurisch uminterpretiert wird. Das futur simple ist damit [...] gar kein Futur, sondern ein Marker perfektiven Aspekts." Meine französischsprachigen Informanten sahen sich allerdings nicht in der Lage, diesen Standpunkt nachzuvollziehen.

26 Mitko (2000: 13). Ähnlich ebd. p. 187. Mitkos Aussage, durch Verwendung von être en train de werde „das im Verb geschilderte Geschehen ins Zentrum der Mitteilung gerückt" (ebd.), gibt den Sachverhalt nicht ganz korrekt wieder.

27 Vgl. Dietrich (1973), besonders Kapitel 4 und 5 (pp. 157-282).

28 Zustimmend z.B. Rohrer (1976: 172); eher skeptisch Wunderli (1975: 232).

29 Klein (1974: 103). Vgl. auch Meier-Brügger (2000: 236): "Die Aktionsart ist [...] eine Eigenschaft der Verbalbedeutung, gehört also in den Bereich des Lexikons und ist somit eine lexikalische Dimension.“ 


\section{Klein, Aspektoppositionen}

30 Vgl. Eisenberg (1999: 108). Eisenberg zitiert die genannten Verben als Beispiele für die durative Aktionsart.

31 Stammerjohann et al. (1975), unter Aktionsart. Die „klassische“ Einteilung der Aktionsarten von Čelakovsky ist weniger stark differenziert. Im einzelnen umfasst sie die Merkmale ,durativ', ,iterativ'/'frequentativ', ,momentan', ,durativ-perfektiv' und ,iterativ-perfektiv'; vgl. Knobloch (1961ff.), unter Aktionsart.

32 Eine derartige Annahme scheint Leskien bewegt zu haben, zur Beschreibung des semantischen Unterschieds zwischen dt. jagen und erjagen die Termini ,imperfektiv' und ,perfektiv' zu verwenden (vgl. Pollak 1960: 34).

33 Beispiele nach Haensch/Lallemand-Rietkötter (1972: 43f.).

34 Vgl. Wandruszka (1976: 88f.). Nach Wandruszka können diese Aktionsarten auf die Merkmale [+ groß/viel] und [+ klein/wenig] zurückgeführt werden. Der Autor verweist u.a. auf die pejorative Komponente, die bei den abgeleiteten Verben häufig mitgegeben ist.

35 Vgl. Ducháček (1966: 163f.), der als Beispiele für die unterschiedlichen Aktionsarten fast nur nicht abgeleitete Verben aufführt.

36 Aus einer derartigen Verwechslung scheint die ablehnende Haltung Eugenio Coserius gegenüber der Agrellschen Trennung von Aspekten und Aktionsarten zu resultieren. Vgl. Coseriu (1976: 87): „Die Anwendung dieses Systems auf die romanischen Sprachen erscheint [...] fraglich, weil diese Aktionsarten im Romanischen nicht anders als durch das Aspektuelle ausgedrückt werden, nämlich durch weitere Bestimmungen des Verbums“.

37 Die iterative Aktionsart wird zuweilen ebenfalls berücksichtigt. Ducháček (1966: 178f.) nennt als Beispiele für betroffene Verben u.a. frz. complimenter, tousser, caresser, aboyer.

38 Die im folgenden zitierten Beispiele sind, von einigen Ausnahmen abgesehen, Klein (1981: 96115) entnommen. $\mathrm{Zu}$ den ausgewerteten Texten vgl. ebd., pp. 246f.

39 In der nach Frequenz geordneten französischen Wortskala von Juilland (Juilland et al. 1970) belegt voir Platz 53; unter den Verben stellt das Wort das sechsthäufigste dar. Regarder nimmt Platz 230 ein.

40 Vgl. z.B. Coseriu (1976: 97), regarder als Beispiel für die ,durative' Aktionsart.

41 Beispielsatz: Je montai sur le pont du navire et regardai Marseille s'écarter (Gide, L'immoraliste, p. 22).

42 Vgl. hierzu Lund (2003) im vorliegenden Band. 